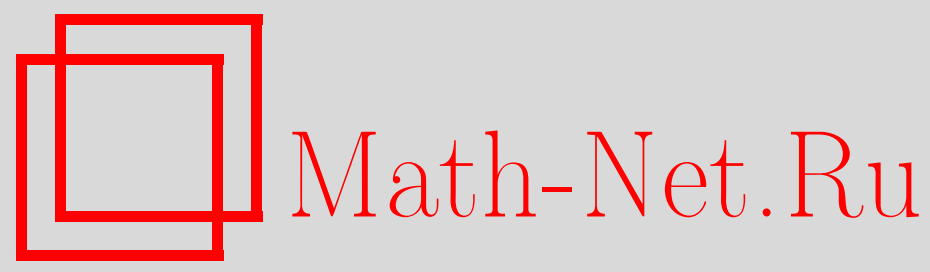

Л. И. Данилов, Об абсолютной непрерывности спектра периодического оператора Шредингера, Матем. заметки, 2003, том 73, выпуск 1, 49-62

DOI: https://doi.org/10.4213/mzm167

Использование Общероссийского математического портала Math-Net.Ru подразумевает, что вы прочитали и согласны с пользовательским соглашением http://www.mathnet.ru/rus/agreement

Параметры загрузки:

IP : 54.89 .56 .158

26 апреля 2023 г., $14: 47: 39$

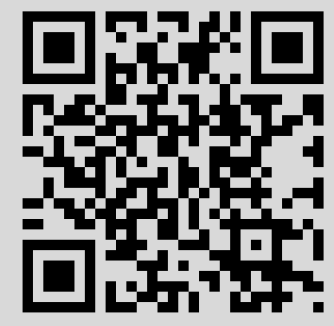




\section{ОБ АБСОЛЮТНОЙ НЕПРЕРЫВНОСТИ СПЕКТРА ПЕРИОДИЧЕСКОГО ОПЕРАТОРА ШРЁДИНГЕРА}

\section{Л.И. Данилов}

Доказана абсолютная непрерывность спектра оператора Шрёдингера в $L^{2}\left(\mathbb{R}^{n}\right)$, $n \geqslant 3$, с периодическими (с общей решеткой периодов $\Lambda$ ) скалярным $V$ и векторным $A \in C^{1}\left(\mathbb{R}^{n}, \mathbb{R}^{n}\right)$ потенциалами, для которых либо $A \in H_{\mathrm{loc}}^{q}\left(\mathbb{R}^{n} ; \mathbb{R}^{n}\right), 2 q>n-2$, либо ряд Фурье векторного потенциала $A$ абсолютно сходится, $V \in L_{w}^{p(n)}(K), K-$ элементарная ячейка решетки $\Lambda, p(n)=n / 2$ при $n=3,4,5,6, p(n)=n-3$ при $n \geqslant 7$, и величина $\lim _{t \rightarrow+\infty}\left\|\theta_{t} V\right\|_{L_{w}^{p(n)}(K)}$ достаточно мала, где $\theta_{t}(x)=0$, если $|V(x)| \leqslant t$, и $\theta_{t}(x)=1$ в противном случае, $x \in K, t>0$.

Библиография: 19 названий.

1. Рассмотрим оператор Шрёдингера

$$
\widehat{H}=\widehat{H}(A)+V=\sum_{j=1}^{n}\left(-i \frac{\partial}{\partial x_{j}}-A_{j}\right)^{2}+V
$$

действующий в $L^{2}\left(\mathbb{R}^{n}\right), n \geqslant 3$. Векторньй (магнитный) и скалярный (электрический) потенциалы $A: \mathbb{R}^{n} \rightarrow \mathbb{R}^{n}$ и $V: \mathbb{R}^{n} \rightarrow \mathbb{R}$ - периодические функции с решеткой периодов $\Lambda \subset \mathbb{R}^{n}$. Пусть

$$
W(A, V ; \phi, \phi)=\sum_{j=1}^{n}\left\|\left(-i \frac{\partial}{\partial x_{j}}-A_{j}\right) \phi\right\|^{2}+(\phi, V \phi)
$$

- соответствуюшая оператору $\widehat{H}(A)+V$ квадратичная форма. Будем предполагать, что вектор-потенциал $A$ непрерывен и форма $(\phi, V \phi)$ имеет грань $<1$ относительно формы $W(0,0 ; \phi, \phi)$ (последнее справедливо, когда потенциал $V$ принадлежит слабому пространству $L_{w}^{n / 2}(K)$, где $K$ - элементарная ячейка решетки $\Lambda$, и величина $\|V\|_{n / 2, w}^{(\infty)}$ достаточно мала). В этом случае форма $W(A, V ; \cdot, \cdot)$ с областью определения $Q(W)=$ $H^{1}\left(\mathbb{R}^{n}\right)$ замкнута и полуограничена снизу. Самосопряженньй оператор (1) с областью определения $D(\widehat{H}) \subset H^{1}\left(\mathbb{R}^{n}\right)$ порождается формой $W(A, V ; \cdot, \cdot)$. Если векторньй потенциал $A$ непрерьвно дифференцируем и потенциал $V$ имеет грань $<1$ относительно оператора $-\Delta$, то самосопряженный оператор $\widehat{H}$ непосредственно определен на функциях $\phi \in D(\widehat{H})=H^{2}\left(\mathbb{R}^{n}\right)$.

Пусть $\Lambda^{*}$ - обратная решетка с базисными векторами $E_{l}^{*}$, удовлетворяюшими условиям $\left(E_{l}^{*}, E_{j}\right)=\delta_{l j}$, где $\left\{E_{j}\right\}$ - базис решетки $\Lambda, v(K)$ и $v\left(K^{*}\right)$ - объемы элементарных ячеек решеток $\Lambda$ и $\Lambda^{*}, v(K) v\left(K^{*}\right)=1, \operatorname{diam} K^{*}$ - диаметр ячейки $K^{*}$. 
Используются стандартные обозначения $C\left(\mathbb{R}^{m} ; \mathbb{C}^{d}\right), C^{l}\left(\mathbb{R}^{m} ; \mathbb{C}^{d}\right)$ и $L^{p}\left(T ; \mathbb{C}^{d}\right)$, где $T=K$ или $T=\mathbb{R}^{m}$, для пространств непрерьвных, непрерывно дифференцируемых до порядка $l$ включительно и с интегрируемой $p$-й степенью нормы (в существенном ограниченных при $p=\infty)$ функций со значениями в $\mathbb{C}^{d}$, через $C_{\Lambda}\left(\mathbb{R}^{n} ; \mathbb{C}^{n}\right)$ и $C_{\Lambda}^{l}\left(\mathbb{R}^{n} ; \mathbb{C}^{n}\right)$ обозначаются соответствующие пространства периодических с решеткой периодов $\Lambda$ функций, $H^{q}\left(\mathbb{R}^{n} ; \mathbb{C}^{d}\right)$ - класс Соболева порядка $q \geqslant 0, \widetilde{H}^{q}\left(K ; \mathbb{C}^{d}\right)$-множество функций $\phi: K \rightarrow \mathbb{C}^{d}$, периодические продолжения которых (с решеткой периодов $\Lambda$ ) принадлежат $H_{\mathrm{loc}}^{q}\left(\mathbb{R}^{n} ; \mathbb{C}^{d}\right)$. Функции, определенные на элементарной ячейке $K$, в дальнейшем будут также отождествляться с их периодическими продолжениями на все пространство $\mathbb{R}^{n}$. Для функций $f: T \rightarrow \mathbb{R}^{d} \subset \mathbb{C}^{d}$ будем в обозначениях заменять $\mathbb{C}^{d}$ на $\mathbb{R}^{d}$ и опускать $\mathbb{C}^{d}$, если $d=1$. Нормы и скалярные произведения $(\cdot, \cdot)$ в пространствах $\mathbb{C}^{d}$, $L^{2}\left(\mathbb{R}^{n} ; \mathbb{C}^{d}\right)$ и $L^{2}\left(K ; \mathbb{C}^{d}\right)$ вводятся обычным образом (без указания в обозначениях самих пространств), при этом предполагается линейность скалярного произведения по второму сомножителю, $|\cdot|$ - норма в $\mathbb{C}^{d},\|\cdot\|$-норма в пространствах $L^{2}\left(\mathbb{R}^{n} ; \mathbb{C}^{d}\right)$ и $L^{2}\left(K ; \mathbb{C}^{d}\right)$.

Через

$$
\chi_{N}=v^{-1}(K) \int_{K} \chi(x) e^{-2 \pi i(N, x)} d x, \quad N \in \Lambda^{*},
$$

обозначаются коэффициенты Фурье функций $\chi \in L^{1}\left(K ; \mathbb{C}^{d}\right)$.

Пусть $\|\cdot\|_{p}$ - норма в пространстве $L^{p}(K), p \geqslant 1\left(\|\cdot\|_{2}=\|\cdot\|\right) ; L_{w}^{p}(K)$ - множество измеримых функций $\mathscr{W}: K \rightarrow \mathbb{C}$, для которых

$$
\|\mathscr{W}\|_{p, w}=\sup _{t>0} t(v(\{x \in K:|\mathscr{W}(x)|>t\}))^{1 / p}<+\infty
$$

где $v(\cdot)$ - мера Лебега. Для $\mathscr{W} \in L_{w}^{p}(K)$ обозначим

$$
\|\mathscr{W}\|_{p, w}^{(\infty)}=\varlimsup_{t \rightarrow+\infty} t(v(\{x \in K:|\mathscr{W}(x)|>t\}))^{1 / p}
$$

$L_{w, 0}^{p}(K)=\left\{\mathscr{W} \in L_{w}^{p}(K):\|\mathscr{W}\|_{p, w}^{(\infty)}=0\right\}$.

Положим $p(n)=n / 2$ при $n=3,4,5,6$ и $p(n)=n-3$ при $n \geqslant 7$. В данной работе доказьвается следующее утверждение.

Tеорема 1. Пусть $n \geqslant 3, A \in C_{\Lambda}^{1}\left(\mathbb{R}^{n} ; \mathbb{R}^{n}\right)$ и либо $\sum_{N \in \Lambda^{*}}\left|A_{N}\right|<+\infty$, либо $A \in H_{\mathrm{loc}}^{q}\left(\mathbb{R}^{n} ; \mathbb{R}^{n}\right)$, әде $2 q>n-2$ (при $n=3$ последнее условие следует из предположсния $\left.A \in C_{\Lambda}^{1}\left(\mathbb{R}^{n} ; \mathbb{R}^{n}\right)\right)$. Тогда найдется число $C^{\prime}=C^{\prime}(n, \Lambda ; A)>0$ такое, что для всех потенииалов $V \in L_{w}^{p(n)}(K ; \mathbb{R})$, для которых $\|V\|_{p(n), w}^{(\infty)}<C^{\prime}$, спектр оператора Шрёдингера (1) абсолютно непрерывен.

Теорема 1 является следствием теоремы 2. При доказательстве используется метод Томаса, предложенньй в [1] для доказательства абсолютной непрерывности спектра оператора Шрёдингера в $L^{2}\left(\mathbb{R}^{3}\right)$ с периодическим электрическим потенциалом $V \in$ $L_{\text {loc }}^{2}\left(\mathbb{R}^{3}\right)$. Метод Томаса используется во многих последующих работах. В [2] результаты работы [1] обобщены на $n$-мерные операторы Шрёдингера с периодическим потенциалом $V$, для которого $V \in L_{\text {loc }}^{2}\left(\mathbb{R}^{n}\right)$ при $n=2,3$ и $\sum_{N \in \Lambda^{*}}\left|V_{N}\right|^{q}<+\infty, 1 \leqslant q<$ $(n-1)(n-2)^{-1}$ при $n \geqslant 4$. В [3] М.Ш. Бирманом и Т. А. Суслиной доказана абсолютная непрерьвность спектра двумерного периодического оператора Шрёдингера в случае $V \in L^{q}(K)$ и $A \in L^{2 q}\left(K ; \mathbb{R}^{2}\right), q>1$. При $n \geqslant 3$ абсолютная непрерьвность спектра 
оператора Шрёдингера была установлена А. Соболевым [4] для периодических потенциалов $V \in L^{q}(K), q>n-1$, и $A \in C_{\Lambda}^{2 n+3}\left(\mathbb{R}^{n} ; \mathbb{R}^{n}\right)$. Это утверждение в дальнейшем было усилено: в [5] предполагается, что $V \in L_{w, 0}^{n / 2}(K)$ при $n=3,4$ и $V \in L_{w, 0}^{n-2}(K)$ при $n \geqslant 5$, и Соболевым было ослаблено условие на векторньй потенциал до условия $A \in H_{\mathrm{loc}}^{q}\left(\mathbb{R}^{n} ; \mathbb{R}^{n}\right), 2 q>3 n-2$ (см. замечание в конце обзорной статьи [5]). Последнее условие на векторньй потенциал $A$ было получено также П. Кучментом и С. Левендорским в работе [6], в которой предложен общий подход к исследованию периодических эллиптических операторов, обобщающий построения Соболева [4] (и доказьвается отсутствие собственных значений в спектре периодического оператора Шрёдингера с комплексными потенциалами $V$ и $A$ ). В [7] доказана абсолютная непрерывность спектра оператора Шрёдингера с периодическим потенциалом $V \in L_{w}^{n / 2}(K)$, для которого величина $\|V\|_{n / 2, w}^{(\infty)}$ достаточно мала, при всех $n \geqslant 3$ (и при $A \equiv 0$ ). Периодические операторы Шрёдингера с переменной метрикой $(n=2)$ и периодический изотропньй оператор Максвелла рассматривались в [8], [5] и [9].

В данной работе результаты для периодического оператора Шрёдингера получены как следствие соответствующих утверждений для периодического оператора Дирака. Ряд условий на электрический потенциал $V$ (при малом вектор-потенциале $A$ ), при которых спектр периодического оператора Дирака абсолютно непрерывен, приведен в [10], [11]. В [12] доказана абсолютная непрерьвность спектра двумерного периодического оператора Дирака при $V \in L_{\mathrm{loc}}^{q}\left(\mathbb{R}^{2}\right)$ и $A \in L_{\mathrm{loc}}^{q}\left(\mathbb{R}^{2} ; \mathbb{R}^{2}\right), q>2$ (при доказательстве используются методы работы [13], в которой рассматривался периодический потенциал $V \in L_{\text {loc }}^{q}\left(\mathbb{R}^{2}\right), q>2$ (при $\left.A \equiv 0\right)$ ). Последнее утверждение получено также (на основе результатов об операторе Шрёдингера) Бирманом и Суслиной [14]. В этой же работе [14] рассмотрен периодический оператор Дирака в $L^{2}\left(\mathbb{R}^{n}, \mathbb{C}^{M}\right), n \geqslant 3$, в случае $V \in C_{\Lambda}\left(\mathbb{R}^{n}\right)$ и при тех же ограничениях на вектор-потенциал $A$, что и для оператора Шрёдингера [5]. В [15], [16] доказана абсолютная непрерьвность спектра оператора Дирака в $L^{2}\left(\mathbb{R}^{n}, \mathbb{C}^{M}\right), n \geqslant 3$, с потенциалами $V \in C_{\Lambda}\left(\mathbb{R}^{n}\right)$ и $A \in C_{\Lambda}\left(\mathbb{R}^{n} ; \mathbb{R}^{n}\right)$, при этом предполагалось, что либо $\sum_{N \in \Lambda^{*}}\left|A_{N}\right|<+\infty$, либо $A \in H_{\mathrm{loc}}^{q}\left(\mathbb{R}^{n} ; \mathbb{R}^{n}\right), 2 q>n-2$.

Часть утверждений настоящей работы содержится в [16], поэтому приводится без доказательств (в [16] накладывались более сильные ограничения на потенциал $V:$ выбиралась функция $p(n)=n / 2$ при $n=3,4,5$ и $p(n)=n^{-1}(n-1)(n-2)$ при $\left.n \geqslant 6\right)$. Без доказательств будет приведен также ряд простых утверждений.

Обозначим $\mathscr{P}_{R}^{\Lambda}=\left\{F \in C_{\Lambda}\left(\mathbb{R}^{n}\right): F(x) \geqslant 0\right.$ при всех $x \in \mathbb{R}^{n}, F_{0}=v^{-1}(K)$ и $F_{N}=0$ при $\left.N \in \Lambda^{*}: 2 \pi|N|>R\right\}, R>0$. Для любых функции $F \in \mathscr{P}_{R}^{\Lambda}, R \geqslant 0$, и вектор-функции $A \in L^{1}\left(K ; \mathbb{C}^{n}\right)$ имеем $F * A \in C_{\Lambda}\left(\mathbb{R}^{n} ; \mathbb{C}^{n}\right)$ и $(F * A)_{N}=v(K) F_{N} A_{N}$, $N \in \Lambda^{*}$, где $(F * A)(x)=\int_{K} F(x-y) A(y) d y, x \in \mathbb{R}^{n}$. Для вектор-функции $A \in$ $C_{\Lambda}\left(\mathbb{R}^{n} ; \mathbb{C}^{n}\right)$ положим

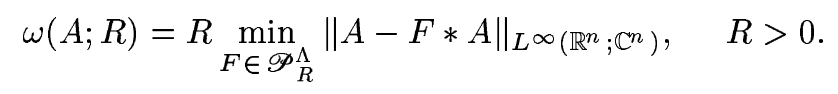

Пусть $\mathscr{B}(\mathbb{R})$ - множество борелевских подмножеств $\mathscr{O} \subset \mathbb{R} ; \mathscr{M}_{h}, h>0,-$ множество знаконеопределенных четных (борелевских) мер $\mu: \mathscr{B}(\mathbb{R}) \rightarrow \mathbb{R}$, для которых

$$
\begin{gathered}
\hat{\mu}(p)=\int_{\mathbb{R}} e^{i p t} d \mu(t)=1 \quad \text { при }|p| \leqslant 2 \pi h, \quad p \in \mathbb{R}, \\
\|\mu\|=\sup _{\mathscr{O} \in \mathscr{B}(\mathbb{R})}(|\mu(\mathscr{O})|+|\mu(\mathbb{R} \backslash \mathscr{O})|)<+\infty, \quad \mu \in \mathscr{M}_{h} .
\end{gathered}
$$


Для любых вектор-функции $A \in C_{\Lambda}\left(\mathbb{R}^{n} ; \mathbb{C}^{n}\right)$, вектора $\gamma \in \Lambda \backslash\{0\}$, меры $\mu \in \mathscr{M}_{h}, h>0$, и вектора $\tilde{e} \in S_{n-2}(e)=\left\{e^{\prime} \in S_{n-1}:\left(e, e^{\prime}\right)=0\right\}$, где $e=|\gamma|^{-1} \gamma\left(S_{n-1}\right.$ - единичная сфера в $\left.\mathbb{R}^{n}\right)$, обозначим

$$
\widetilde{A}(\gamma, \mu, \tilde{e} ; x)=\int_{\mathbb{R}} d \mu(t) \int_{0}^{1} A(x-\xi \gamma-t \tilde{e}) d \xi, \quad x \in \mathbb{R}^{n}
$$

$\tilde{A}(\gamma, \mu, \tilde{e} ; \cdot) \in C_{\Lambda}\left(\mathbb{R}^{n} ; \mathbb{C}^{n}\right)$.

Теорема 2. Пусть $n \geqslant 3, \Lambda$ - решетка в $\mathbb{R}^{n}$. Предположим, что для векторного потенииала $A \in C_{\Lambda}\left(\mathbb{R}^{n} ; \mathbb{R}^{n}\right)$ найдутся вектор $\gamma \in \Lambda \backslash\{0\}, e=|\gamma|^{-1} \gamma$, и мера $\mu \in \mathscr{M}_{h}, h>0$, такие, что

$$
\max _{\tilde{e} \in S_{n-2}(e)}\left\|\widetilde{A}(\gamma, \mu, \tilde{e} ; \cdot)-A_{0}\right\|_{L^{\infty}\left(\mathbb{R}^{n} ; \mathbb{R}^{n}\right)}=\theta \pi|\gamma|^{-1}
$$

где $\theta \in[0,1), A_{0}=v^{-1}(K) \int_{K} A(x) d x$, и для некоторого числа $R \geqslant \pi|\gamma|^{-1}$ вьиолнено неравенство

$$
|\gamma|^{2} \omega(A ; R) \leqslant C_{2}^{\prime}(1-\theta)^{18},
$$

әде $C_{2}^{\prime}=C_{1} C_{0}(A), C_{0}(A)=\exp \left\{-C h^{-1}\|\mu\|\|A\|_{L^{\infty}\left(\mathbb{R}^{n} ; \mathbb{R}^{n}\right)}\right\}, C>0-$ универсальная постоянная, $C_{1}=C_{1}(n) \in(0,1]$. Предположим также, ито $V \in L_{w}^{p(n)}(K ; \mathbb{R}) u$

$$
\|V\|_{p(n), w}^{(\infty)}<C_{3}^{-1} C_{2}^{\prime}|\gamma|^{-2} R^{-2}(1-\theta)^{18}
$$

где $C_{3}=1$ при $n=3,4,5$ и $C_{3}=\left(\operatorname{diam} K^{*}\right)^{-(n-6) /(n-3)}\left(1+|\gamma| \operatorname{diam} K^{*}\right) n p u n \geqslant 6$. Тогда спектр оператора Шрёдингера (1) абсолютно непрерывен.

ЗАмЕЧАнИЕ. Условие (4) предполагает (при соответствующем выборе числа $C_{1}$ ), что форма $(\phi, V \phi)$ имеет грань $<1$ относительно формы $W(0,0 ; \phi, \phi), \phi \in H^{1}\left(\mathbb{R}^{n}\right)$.

При доказательстве теоремы 2 (переходя в случае необходимости к оператору $\left.e^{-i\left(A_{0}, x\right)} \widehat{H} e^{i\left(A_{0}, x\right)}\right)$ можно считать, что $A_{0}=0$.

Если $A \in C_{\Lambda}\left(\mathbb{R}^{n} ; \mathbb{R}^{n}\right)$ и либо $\sum_{N \in \Lambda^{*}}\left|A_{N}\right|<+\infty$, либо $A \in H_{\mathrm{loc}}^{q}\left(\mathbb{R}^{n} ; \mathbb{R}^{n}\right), 2 q>n-2$, то найдутся вектор $\gamma \in \Lambda \backslash\{0\}$ и мера $\mu \in \mathscr{M}_{h}, h \in\left(0,|\gamma|^{-1}\right]$, такие, что для некоторого $\theta \in[0,1)$ выполняется условие (2) [15], [16]. Если $A \in C_{\Lambda}^{1}\left(\mathbb{R}^{n} ; \mathbb{R}^{n}\right)$, то $\omega(A ; R) \rightarrow 0$ при $R \rightarrow+\infty$ и, следовательно, неравенство (3) справедливо для всех достаточно больших $R$. Поэтому теорема 1 непосредственно вытекает из теоремы 2 .

2. Пусть $k \in \mathbb{R}^{n}, \kappa \in \mathbb{R}, e \in S_{n-1}, A \in C_{\Lambda}\left(\mathbb{R}^{n} ; \mathbb{C}^{n}\right)$,

$W(A ; k+i \kappa e ; \psi, \phi)=\sum_{j=1}^{n}\left(\left(-i \frac{\partial}{\partial x_{j}}-\bar{A}_{j}+k_{j}-i \kappa e_{j}\right) \psi,\left(-i \frac{\partial}{\partial x_{j}}-A_{j}+k_{j}+i \kappa e_{j}\right) \phi\right)$

- полуторалинейная форма с областью определения $Q(W)=\widetilde{H}^{1}(K) \subset L^{2}(K)$. Если $V \in L_{w}^{n / 2}(K ; \mathbb{C})$ и $\|V\|_{n / 2, w}^{(\infty)}<C_{1}^{\prime}$ для некоторого (достаточно малого) $C_{1}^{\prime}=C_{1}^{\prime}(n)>0$, то форма $(\phi, V \phi)$ имеет грань $<1$ относительно формы $W(0 ; k ; \phi, \phi)$. В этом случае

$$
W(V, A ; k+i \kappa e ; \phi, \phi)=W(A ; k+i \kappa e ; \phi, \phi)+(\phi, V \phi), \quad \phi \in \widetilde{H}^{1}(K),
$$


- замкнутая и секториальная квадратичная форма, порождающая $m$-секториальный оператор $\widehat{H}(A ; k+i \kappa e)+V$. Если $A \in C_{\Lambda}^{1}\left(\mathbb{R}^{n} ; \mathbb{C}^{n}\right)$, то

$$
\widehat{H}(A ; k+i \kappa e)=\sum_{j=1}^{n}\left(-i \frac{\partial}{\partial x_{j}}-A_{j}+k_{j}+i \kappa e_{j}\right)^{2}
$$

$D(\widehat{H}(A ; k+i \kappa e))=\widetilde{H}^{2}(K)$.

Оператор (1) унитарно эквивалентен прямому интегралу

$$
\int_{2 \pi K^{*}} \oplus(\widehat{H}(A ; k)+V) \frac{d k}{(2 \pi)^{n} v\left(K^{*}\right)} .
$$

Унитарная эквивалентность устанавливается с помощью преобразования Гельфанда [3], [7]. Операторы $\widehat{H}(A ; k)+V$ имеют компактную резольвенту и, следовательно, дискретный спектр. Для доказательства абсолютной непрерьвности спектра оператора (1) достаточно показать, что существует вектор $e \in S_{n-1}$ такой, что для любых $E \in \mathbb{R}$ и $k \in 2 \pi K^{*}$ найдется число $\xi+i \kappa \in \mathbb{C}$, для которого оператор $\widehat{H}(A ; k+(\xi+i \kappa) e)+V-E$ обратим (подробное доказательство этого утверждения приведено в [17], [2], [3], [7]), поэтому теорема 2 является следствием теоремы 3, в которой установлена обратимость операторов $\widehat{H}\left(A ; k+i \kappa|\gamma|^{-1} \gamma\right)+V-E$ для некоторых вектора $\gamma \in \Lambda \backslash\{0\}$ и достаточно больших $\kappa$ и при всех $k \in \mathbb{R}^{n}:(k, \gamma)=\pi$.

Выберем (и зафиксируем) векторы $\gamma \in \Lambda \backslash\{0\}, e=|\gamma|^{-1} \gamma \in S_{n-1}$. Для всех $N \in \Lambda^{*}$, $k \in \mathbb{R}^{n}$ и $\kappa \in \mathbb{R}$ обозначим

$$
G_{N}^{ \pm}=G_{N}^{ \pm}(k ; \kappa)=\left((k+2 \pi N, e)^{2}+\left(|\kappa| \pm \sqrt{|k+2 \pi N|^{2}-(k+2 \pi N, e)^{2}}\right)^{2}\right)^{1 / 2}
$$

$G_{N}=G_{N}(k ; \kappa)=G_{N}^{-}(k ; \kappa)$. Справедливо равенство

$$
\widehat{H}(0 ; k+i \kappa e) \phi=\sum_{N \in \Lambda^{*}}(k+2 \pi N+i \kappa e)^{2} \phi_{N} e^{2 \pi i(N, x)}, \quad \phi \in \widetilde{H}^{2}(K),
$$

при этом $\left|(k+2 \pi N+i \kappa e)^{2}\right|=G_{N} G_{N}^{+}$. Если $(k, \gamma)=\pi$, то $G_{N} \geqslant \pi|\gamma|^{-1}$ и $G_{N} G_{N}^{+} \geqslant$ $2 \pi|\gamma|^{-1}|\kappa|$. Пусть $\widehat{L}=\widehat{L}(k ; \kappa)$ - неотрицательный оператор в $L^{2}(K)$ :

$$
\widehat{L} \phi=\sum_{N \in \Lambda^{*}} G_{N} G_{N}^{+} \phi_{N} e^{2 \pi i(N, x)}, \quad \phi \in D(\widehat{L})=\widetilde{H}^{2}(K) .
$$

Будем обозначать $\|\phi\|_{*}=\left\|\widehat{L}^{1 / 2} \phi\right\|, \phi \in D\left(\widehat{L}^{1 / 2}\right)=\widetilde{H}^{1}(K)$.

Tеорема 3. Пусть $n \geqslant 3, \gamma \in \Lambda \backslash\{0\}, e=|\gamma|^{-1} \gamma, \mu \in \mathscr{M}_{h}, h>0, \theta \in[0,1)$, $Y_{0} \geqslant 0$ и $R \geqslant \pi|\gamma|^{-1}$. Тогда существуют универсальная постоянная $C>0$, числа $C_{1}=C_{1}(n)>0 u C_{3}=1$ npu $n=3,4,5, C_{3}=\left(\operatorname{diam} K^{*}\right)^{-(n-6) /(n-3)}\left(1+|\gamma| \operatorname{diam} K^{*}\right)$ при $n \geqslant 6$ такие, что для всех вектор-функиий $A \in C_{\Lambda}\left(\mathbb{R}^{n} ; \mathbb{C}^{n}\right)$, для которых $A_{0}=0$ и выполнены условия

$$
\begin{gathered}
\|A\|_{L^{\infty}\left(\mathbb{R}^{n} ; \mathbb{C}^{n}\right)} \leqslant Y_{0} \\
\max _{\tilde{e} \in S_{n-2}(e)}\|(\widetilde{A}(\gamma, \mu, \widetilde{e} ; \cdot), \widetilde{e})+i(\widetilde{A}(\gamma, \mu, \widetilde{e} ; \cdot), e)\|_{L^{\infty}\left(\mathbb{R}^{n}\right)} \leqslant \theta \pi|\gamma|^{-1} \\
|\gamma|^{2} \omega(A ; R) \leqslant C_{2}(1-\theta)^{18}
\end{gathered}
$$


$2 \partial e$

$$
C_{2}=C_{1} \exp \left(-C h^{-1}\|\mu\| Y_{0}\right)
$$

любой функиии $V \in L_{w}^{p(n)}(K ; \mathbb{C})$, для которой

$$
\|V\|_{p(n), w}^{(\infty)}<C_{3}^{-1} C_{2}|\gamma|^{-2} R^{-2}(1-\theta)^{18}
$$

и любого числа $E \in \mathbb{C}$ найдется такое число $\kappa>0$, что для всех $k \in \mathbb{R}^{n}:(k, \gamma)=\pi$ и всех ненулевых функиий $\phi \in \widetilde{H}^{1}(K)$ справедливо неравенство

$$
\sup _{\psi \in \widetilde{H}^{1}(K):\|\psi\|_{*} \leqslant 1}|W(V-E, A ; k+i \kappa e ; \psi, \phi)|>0
$$

$u$, следовательно, оператор $\widehat{H}(A ; k+i \kappa e)+V-E$ обратим.

ЗАмЕчАниЕ. Оператор (1) можно рассматривать с комплекснозначными потенциалами $V$ и $A$. В этом случае из теоремы 3 и разложения (5) следует, что в спектре оператора $\widehat{H}(A)+V$ (при соответствуюших ограничениях на $V$ и $A$ ) отсутствуют собственные значения. В частности, это справедливо, если потенциалы $V$ и $A$ удовлетворяют условиям теоремы 1 (которые были сформулированы для вешественнозначных потенциалов).

Теорема 3 является следствием теорем 4 и 5.

Teopema 4. Пyсmb $n \geqslant 3, \gamma \in \Lambda \backslash\{0\}, e=|\gamma|^{-1} \gamma, \mu \in \mathscr{M}_{h}, h>0, \theta \in[0,1)$, $Y_{0} \geqslant 0$ и $R \geqslant \pi|\gamma|^{-1}$. Тогда существуют универсальная постоянная $C>0 u$ число $C_{1}=C_{1}(n) \in(0,1]$ (для них постоянная $C_{2}$ определяется по формуле $(9)$ ) такие, что для любой вектор-функиии $A \in C_{\Lambda}\left(\mathbb{R}^{n} ; \mathbb{C}^{n}\right)$, для которой $A_{0}=0$ и выполнены условия (6)-(8), найдется число $\kappa_{0}>0$ такое, что для всех $\kappa \geqslant \kappa_{0}$, всех векторов $k \in \mathbb{R}^{n}:(k, \gamma)=\pi$ и всех функиий $\phi \in \widetilde{H}^{1}(K)$ справедливо неравенство

$$
\sup _{\psi \in \widetilde{H}^{1}(K):\|\psi\|_{*} \leqslant 1}|W(A ; k+i \kappa e ; \psi, \phi)| \geqslant C_{2}|\gamma|^{-2} R^{-2}(1-\theta)^{18}\|\phi\|_{*} .
$$

Tеорема 5. Пусть $n \geqslant 3, \gamma \in \Lambda \backslash\{0\}, e=|\gamma|^{-1} \gamma, \epsilon>0$. Тогда для всех функиий $\mathscr{W} \in L_{w}^{2 p(n)}(K ; \mathbb{C})$, всех векторов $k \in \mathbb{R}^{n}:(k, \gamma)=\pi$, всех $\kappa \geqslant \kappa_{0}=\kappa_{0}(n, \Lambda, \gamma ; \epsilon, \mathscr{W})$ $>0$ при $n \neq 4$ и для некоторой последовательности $\kappa=\kappa_{\nu} \geqslant \kappa_{0}, \nu \in \mathbb{N}$, для которой $\kappa_{\nu} \rightarrow+\infty$ nри $\nu \rightarrow+\infty$, при $n=4$ и для всех функиий $\phi \in \widetilde{H}^{1}(K)$ справедливо неравенство

$$
\|\mathscr{W} \phi\| \leqslant \widetilde{C}_{3}\left(\epsilon+\|\mathscr{W}\|_{2 p(n), w}^{(\infty)}\right)\|\phi\|_{*},
$$

әде $\widetilde{C}_{3}>0$ - некоторая универсальная постоянная при $n=3,4,5$ и $\widetilde{C}_{3}=\widetilde{C}_{3}^{\prime}(n) \times$ $\left(\operatorname{diam} K^{*}\right)^{-(n-6) /(2(n-3))}\left(1+\left(|\gamma| \operatorname{diam} K^{*}\right)^{1 / 2}\right)>0$ npu $n \geqslant 6$. 
3. Пусть $\widehat{\alpha}_{j}, j=1, \ldots, n,-$ эрмитовы $(M \times M)$-матрицы, удовлетворяющие антикоммутационным соотношениям $\hat{\alpha}_{j} \hat{\alpha}_{l}+\hat{\alpha}_{l} \hat{\alpha}_{j}=2 \delta_{j l} \hat{I}_{M}$, где $\hat{I}_{M}$ - единичная $(M \times M)$-матрица (можно считать, что $\left.M=2^{[n / 2]}\right), A \in C_{\Lambda}\left(\mathbb{R}^{n} ; \mathbb{C}^{n}\right)$,

$$
\widehat{D}(A ; k+i \kappa e)=\sum_{j=1}^{n} \hat{\alpha}_{j}\left(-i \frac{\partial}{\partial x_{j}}-A_{j}+k_{j}+i \kappa e_{j}\right)
$$

- оператор Дирака с областью определения $D(\widehat{D}(A ; k+i \kappa e))=\widetilde{H}^{1}\left(K ; \mathbb{C}^{M}\right) \subset$ $L^{2}\left(K ; \mathbb{C}^{M}\right)$. Если $A \in C_{\Lambda}^{1}\left(\mathbb{R}^{n} ; \mathbb{C}^{n}\right)$, то

$$
\widehat{D}^{2}(A ; k+i \kappa e)=\widehat{H}(A ; k+i \kappa e) \otimes \hat{I}_{M}+\frac{i}{2} \sum_{j, l=1}^{n}\left(\frac{\partial A_{l}}{\partial x_{j}}-\frac{\partial A_{j}}{\partial x_{l}}\right) \hat{\alpha}_{j} \hat{\alpha}_{l}
$$

$D\left(\widehat{D}^{2}(A ; k+i \kappa e)\right)=\widetilde{H}^{2}\left(K ; \mathbb{C}^{M}\right)$.

Для $b>0$ при $\kappa \in \mathbb{R}:|\kappa|>b$ обозначим

$$
\begin{gathered}
\mathscr{O}_{b}=\mathscr{O}_{b}(\kappa)=\left\{x \in \mathbb{R}^{n}:(x, e)^{2}+(|\kappa|-|x-(x, e) e|)^{2}<b^{2}\right\}, \\
\mathscr{K}_{b}=\mathscr{K}_{b}(k ; \kappa)=\left\{N \in \Lambda^{*}: k+2 \pi N \in \mathscr{O}_{b}\right\}, \quad k \in \mathbb{R}^{n} .
\end{gathered}
$$

Через $\widehat{P}^{\mathscr{C}}$, где $\mathscr{C} \subset \Lambda^{*}$, обозначим ортогональный проектор в пространстве $L^{2}(K)$, ставящий в соответствие функциям $\phi \in L^{2}(K)$ функции $\widehat{P}^{\mathscr{C}} \phi=\phi^{\mathscr{C}}=\sum_{N \in \mathscr{C}} \phi_{N} e^{2 \pi i(N, x)}$ $\left(\phi^{\varnothing} \equiv 0\right) ; \mathscr{H}_{d}(\mathscr{C})=\left\{\phi \in L^{2}\left(K ; \mathbb{C}^{d}\right): \phi_{N}=0\right.$ при $\left.N \notin \mathscr{C}\right\} ; \mathscr{H}(\mathscr{C})=\mathscr{H}_{1}(\mathscr{C})$.

Доказательство следующей теоремы приведено в [16] (вариант этой теоремы без явного определения оценочной постоянной в неравенстве (12) содержится также в [15]). Из теоремы 6 и равенства (10) следуют теоремы 7 и 8 [16].

Tеорема 6. Пусть (при $n \geqslant 3$ и при фиксированных векторе $\gamma \in \Lambda \backslash\{0\}$ и мере $\left.\mu \in \mathscr{M}_{h}, h \in\left(0,|\gamma|^{-1}\right], e=|\gamma|^{-1} \gamma\right) \quad \theta \in[0,1), Y_{0} \geqslant|\gamma|^{-1}, R \geqslant \pi|\gamma|^{-1}$ $u b>0$. Тогда существуют число $\kappa_{0}>0$, универсальная постоянная $C>0$ и число $C_{1}=C_{1}(n) \in(0,1]$ (постоянная $C_{2}$ выражсается через них по формуле (9)) такие, что для всех $\kappa \geqslant \kappa_{0}$, всех векторов $k \in \mathbb{R}^{n}:(k, \gamma)=\pi$, всех вектор-функиий $A \in C_{\Lambda}\left(\mathbb{R}^{n} ; \mathbb{C}^{n}\right)$, для которых $A_{0}=0$ и выполнены условия (6), (7) и

$$
A_{N}=0 \quad \forall N \in \Lambda^{*}: 2 \pi|N|>R,
$$

и всех вектор-функиий $\phi \in \mathscr{H}_{M}\left(\mathscr{K}_{b}(k ; \kappa)\right)$ справедливо неравенство

$$
\left\|\widehat{D}^{2}(A ; k+i \kappa e) \phi\right\| \geqslant C_{2}|\gamma|^{-2}(1-\theta)^{6} \frac{\kappa}{b+R}\|\phi\| .
$$


TEорема 7. Пусть (при $n \geqslant 3$ и при фиксированных векторе $\gamma \in \Lambda \backslash\{0\}$ и мере $\left.\mu \in \mathscr{M}_{h}, h \in\left(0,|\gamma|^{-1}\right], e=|\gamma|^{-1} \gamma\right) \quad \theta \in[0,1), Y_{0} \geqslant|\gamma|^{-1}, Y_{1} \geqslant 0, R \geqslant \pi|\gamma|^{-1}$ $u b>0$. Тогда существуют число $\kappa_{0}>0$, универсальная постоянная $C>0 u$ иисло $C_{1}=C_{1}(n) \in(0,1]$ такие, что для всех $\kappa \in \mathbb{R}:|\kappa| \geqslant \kappa_{0}$, всех векторов $k \in \mathbb{R}^{n}:(k, \gamma)=\pi$, всех вектор-функиий $A \in C_{\Lambda}^{1}\left(\mathbb{R}^{n} ; \mathbb{C}^{n}\right)$, для которых $A_{0}=0 u$ выполнены условия (6), (7), (11) и

$$
\left\|\frac{\partial A_{j}}{\partial x_{l}}\right\|_{L^{\infty}\left(\mathbb{R}^{n}\right)} \leqslant Y_{1}, \quad j, l=1, \ldots, n
$$

и всех функиий $\phi \in \mathscr{H}\left(\mathscr{K}_{b}(k ; \kappa)\right)$ справедливо неравенство

$$
\|\widehat{H}(A ; k+i \kappa e) \phi\| \geqslant C_{2}|\gamma|^{-2}(1-\theta)^{6} \frac{|\kappa|}{b+R}\|\phi\|,
$$

где $C_{2}$ определена в (9).

Tеорема 8. Пусть (при $n \geqslant 3$ и при фиксированных векторе $\gamma \in \Lambda \backslash\{0\}$ и мере $\left.\mu \in \mathscr{M}_{h}, h \in\left(0,|\gamma|^{-1}\right], e=|\gamma|^{-1} \gamma\right) \quad \theta \in[0,1), Y_{0} \geqslant 0, Y_{1} \geqslant 0 u R \geqslant \pi|\gamma|^{-1}$. Тогдa существуют число к $\kappa_{0}>0$, универсальная постоянная $C>0$ и число $C_{1}=C_{1}(n) \in$ $(0,1]$ (постоянная $C_{2}$ определяется через них по формуле (9)) такие, что для всех $\kappa \in \mathbb{R}:|\kappa| \geqslant \kappa_{0}$, всех векторов $k \in \mathbb{R}^{n}:(k, \gamma)=\pi$, всех вектор-функиий $A \in C_{\Lambda}^{1}\left(\mathbb{R}^{n} ; \mathbb{C}^{n}\right)$, для которых $A_{0}=0$ и выполнены условия (6)-(8) и (13), и всех функиий $\phi \in \widetilde{H}^{2}(K)$ справедливо неравенство

$$
\|\widehat{H}(A ; k+i \kappa e) \phi\| \geqslant C_{2}|\gamma|^{-2} R^{-2}(1-\theta)^{18}\|\widehat{H}(0 ; k+i \kappa e) \phi\| .
$$

ДокаЗАТЕЛЬСТво тЕОРемЫ 4. Пусть вначале $A \in C_{\Lambda}^{1}\left(\mathbb{R}^{n} ; \mathbb{C}^{n}\right), A_{0}=0$ и вьполнены условия (6)-(8);

$$
Y_{1}=\max _{j, l=1, \ldots, n}\left\|\frac{\partial A_{j}}{\partial x_{l}}\right\|_{L^{\infty}\left(\mathbb{R}^{n}\right)},
$$

$C, C_{1}$ и $\kappa_{0}-$ постоянные из теоремы 8 ( $\kappa_{0}$ зависит от $Y_{1}$ и, следовательно, от выбора вектор-функции $A$ ). Обозначим $\widetilde{C}_{2}=C_{2}|\gamma|^{-2} R^{-2}(1-\theta)^{18}$. Из теоремы 8 следует, что при всех $\kappa \in \mathbb{R}:|\kappa| \geqslant \kappa_{0}$ и всех $k \in \mathbb{R}^{n}:(k, \gamma)=\pi$ оператор $\widehat{H}(A ; k+i \kappa e)$ (как и оператор $\widehat{H}(0 ; k+i \kappa e))$ обратим, $D(\widehat{H}(A ; k+i \kappa e))=D(\widehat{H}(0 ; k+i \kappa e))=\widetilde{H}^{2}(K)$ и $L^{2}(K)$ - его область значений. При этом $\left\|\widehat{H}(0 ; k+i \kappa e) \widehat{H}^{-1}(A ; k+i \kappa e)\right\| \leqslant \widetilde{C}_{2}^{-1}$. Оператор $\widehat{H}^{-1}(A ; k+i \kappa e) \widehat{H}(0 ; k+i \kappa e)$ непрерывно продолжается с линейного многообразия $\widetilde{H}^{2}(K) \subset L^{2}(K)$ на все пространство $L^{2}(K)$ и также

$$
\left\|\widehat{H}^{-1}(A ; k+i \kappa e) \widehat{H}(0 ; k+i \kappa e)\right\|=\left\|\widehat{H}(0 ; k-i \kappa e) \widehat{H}^{-1}(\bar{A} ; k-i \kappa e)\right\| \leqslant \widetilde{C}_{2}^{-1} .
$$

Следовательно, $\left\|\widehat{L} \widehat{H}^{-1}(A ; k+i \kappa e)\right\| \leqslant \widetilde{C}_{2}^{-1}$ и $\left\|\widehat{H}^{-1}(A ; k+i \kappa e) \widehat{L}\right\| \leqslant \widetilde{C}_{2}^{-1}$. Используя интерполяцию $[18$, дополнение к $\S \mathrm{IX} .4]$, отсюда получаем

$$
\left\|\widehat{L}^{1 / 2} \widehat{H}^{-1}(A ; k+i \kappa e) \widehat{L}^{1 / 2}\right\| \leqslant \widetilde{C}_{2}^{-1},
$$


поэтому для всех $\widetilde{\phi} \in \widetilde{H}^{1}(K)$ справедлива оценка $\left\|\widehat{L}^{-1 / 2} \widehat{H}(A ; k+i \kappa e) \widehat{L}^{-1 / 2} \widetilde{\phi}\right\| \geqslant \widetilde{C}_{2}\|\widetilde{\phi}\|$. Пусть $\phi \in \widetilde{H}^{2}(K)$. Тогда

$$
\begin{aligned}
& \sup _{\psi \in \widetilde{H}^{1}(K):\|\psi\|_{*} \leqslant 1}|W(A ; k+i \kappa e ; \psi, \phi)|=\sup _{\psi \in \widetilde{H}^{1}(K):\|\psi\|_{*} \leqslant 1}|(\psi, \widehat{H}(A ; k+i \kappa e) \phi)| \\
& =\left\|\widehat{L}^{-1 / 2} \widehat{H}(A ; k+i \kappa e) \widehat{L}^{-1 / 2}\left(\widehat{L}^{1 / 2} \phi\right)\right\| \geqslant \widetilde{C}_{2}\|\phi\|_{*} .
\end{aligned}
$$

Так как линейное многообразие $\widetilde{H}^{2}(K)$ плотно в $\left(\widetilde{H}^{1}(K),\|\cdot\|_{*}\right)$, а форма $W(A ; k+i \kappa e ;$ $\cdot, \cdot)$ непрерьвна на $\left(\widetilde{H}^{1}(K),\|\cdot\|_{*}\right)$, то

$$
\sup _{\psi \in \widetilde{H}^{1}(K):\|\psi\|_{*} \leqslant 1}|W(A ; k+i \kappa e ; \psi, \phi)| \geqslant \widetilde{C}_{2}\|\phi\|_{*}
$$

для всех $\phi \in \widetilde{H}^{1}(K)$. Пусть теперь $A \in C_{\Lambda}\left(\mathbb{R}^{n} ; \mathbb{C}^{n}\right), A_{0}=0$ и вьполнены условия (6)-(8). Апшроксимируя в $L^{\infty}\left(\mathbb{R}^{n} ; \mathbb{C}^{n}\right)$ вектор-функцию $A$ тригонометрическими многочленами $F * A \in C_{\Lambda}^{1}\left(\mathbb{R}^{n} ; \mathbb{C}^{n}\right)$, где $F \in \mathscr{P}_{r}^{\Lambda}$ и $r \rightarrow+\infty$ (для которых $(F * A)_{0}=0$ и вьполнены условия (6)-(8)), учитывая, что при $\kappa \geqslant \max \left\{1, \pi|\gamma|^{-1}\right\}$

$$
\begin{aligned}
& |W(A ; k+i \kappa e ; \psi, \phi)-W(F * A ; k+i \kappa e ; \psi, \phi)| \\
& \quad \leqslant 2\left(4+n Y_{0}\right) \pi^{-1}|\gamma|\|A-F * A\|_{L^{\infty}\left(\mathbb{R}^{n} ; \mathbb{C}^{n}\right)}\|\psi\|_{*}\|\phi\|_{*},
\end{aligned}
$$

и уменьшая числа $C_{1}$ и $C_{2}$ (выбирая вместо них, например, числа $\frac{1}{2} C_{1}$ и $\left.\frac{1}{2} C_{2}\right)$, из (14) (при $\kappa \geqslant \kappa_{0}$ для некоторого $\kappa_{0}>0$, зависящего от вектор-функции $A$, и при всех $\left.k \in \mathbb{R}^{n}:(k, \gamma)=\pi\right)$ получаем доказываемое неравенство. Теорема доказана.

4. ДоКАЗАТЕЛЬСТво тЕОРЕмЫ 5. Из интерполяционных теорем Ханта и Марцинкевича следует, что сушествует константа $c^{\prime}=c^{\prime}(n)>0$ такая, что для любых функций $\mathscr{W} \in L_{w}^{n}\left(\mathbb{R}^{n}\right), \mathscr{G} \in L_{w}^{n /(n-1)}\left(\mathbb{R}^{n}\right)$ (из слабых пространств), $n \geqslant 3$, и $\phi \in L^{2}\left(\mathbb{R}^{n}\right)$ функция $\mathscr{W}(\mathscr{G} * \phi)\left(\right.$ где $*-$ свертка в $\left.\mathbb{R}^{n}\right)$ принадлежит пространству $L^{2}\left(\mathbb{R}^{n}\right)$ и

$$
\|\mathscr{W}(\mathscr{G} * \phi)\|_{L^{2}\left(\mathbb{R}^{n}\right)} \leqslant c^{\prime}\|\mathscr{W}\|_{L_{w}^{n}\left(\mathbb{R}^{n}\right)}\|\mathscr{G}\|_{L_{w}^{n /(n-1)}\left(\mathbb{R}^{n}\right)}\|\phi\|_{L^{2}\left(\mathbb{R}^{n}\right)}
$$

Отсюда (см. также [19, глава 5 , тождество (18)]) следует, что найдутся постоянные $c^{\prime \prime}=$ $c^{\prime \prime}(n)>0$ и $c_{1}^{\prime \prime}=c_{1}^{\prime \prime}(n, \Lambda)>0$ такие, что для всех функций $\mathscr{W} \in L_{w}^{n}(K)$ и $\phi \in \widetilde{H}^{1}(K)$

$$
\|\mathscr{W} \phi\| \leqslant\|\mathscr{W}\|_{n, w}\left(c^{\prime \prime} \sum_{j=1}^{n}\left\|\frac{\partial \phi}{\partial x_{j}}\right\|+c_{1}^{\prime \prime}\|\phi\|\right) .
$$

Так как $|k+2 \pi N|^{2} \leqslant 9 G_{N} G_{N}^{+}$для всех $N \in \Lambda^{*} \backslash \mathscr{K}_{\kappa / 8}(k ; \kappa), k \in \mathbb{R}^{n}, \kappa>0$, следующая теорема вытекает из (15).

Теорема 9. При $n \geqslant 3$ (и при фиксированном векторе $\left.\gamma \in \Lambda \backslash\{0\}, e=|\gamma|^{-1} \gamma\right)$ найдутся постоянные $c=c(n)>0 u c_{1}=c_{1}(n, \Lambda)>0$ такие, что для всех $\kappa>0$, всех векторов $k \in \mathbb{R}^{n}$, всех функиий $\mathscr{W} \in L_{w}^{n}(K) u \phi \in \widetilde{H}^{1}(K) \cap \mathscr{H}\left(\Lambda^{*} \backslash \mathscr{K}_{\kappa / 8}(k ; \kappa)\right)$ справедливо неравенство

$$
\|\mathscr{W} \phi\| \leqslant\|\mathscr{W}\|_{n, w}\left(c\|\phi\|_{*}+c_{1}\|\phi\|\right)
$$


Лемма 1. Пусть $\mathscr{W} \in L_{w}^{p}(K), p>2$. Обозначим $K^{(s)}=\{x \in K:|\mathscr{W}(x)|>s\}$, $s>0$. Тогда

$$
\int_{K^{(s)}}|\mathscr{W}(x)|^{2} d x \leqslant c_{4} s^{-(p-2)}\|\mathscr{W}\|_{p, w}^{p}
$$

əде $c_{4}=c_{4}(p)>0$.

Будем далее обозначать $a=2 \pi \operatorname{diam} K^{*}$.

Лемма 2. Пусть $n \geqslant 3, p>2$ (и фиксирован вектор $\left.\gamma \in \Lambda \backslash\{0\}, e=|\gamma|^{-1} \gamma\right)$. Тогда найдется постоянная $c_{5}=c_{5}(n, p)>0$ такая, что для всех $\kappa>2 a, b \in[a, \kappa / 2)$, всех векторов $k \in \mathbb{R}^{n}$ и всех функиий $\mathscr{W} \in L_{w}^{p}(K)$ и $\phi \in \mathscr{H}\left(\mathscr{K}_{b}(k ; \kappa)\right)$ справедливо неравенство

$$
\|\mathscr{W} \phi\| \leqslant c_{5}\|\mathscr{W}\|_{p, w} b^{2 / p} \kappa^{(n-2) / p}\|\phi\|
$$

Так как при $\kappa>2 a, b \in[a, \kappa / 2)$ множество $\mathscr{K}_{b}$ содержит не более $c_{6} v(K) b^{2} \kappa^{n-2}$ векторов, где $c_{6}=c_{6}(n)>0$, лемма 2 вытекает с помощью разбиения функции $\mathscr{W}$ на большую и малую части [19, глава 1] из леммы 1.

Пусть $\kappa \geqslant \kappa_{0}>8 a$. Будем выбирать число $L=L(\kappa) \in \mathbb{N}$ так, что $\kappa / 8 \leqslant 2^{L} a<\kappa / 4$. Пусть

$$
\mathscr{K}_{a}^{0}=\mathscr{K}_{a}, \quad \mathscr{K}_{a}^{j}=\mathscr{K}_{2^{j} a} \backslash \mathscr{K}_{2^{j-1} a}, \quad j=1, \ldots, L .
$$

Для любой функции $\phi \in \mathscr{H}\left(\mathscr{K}_{2^{L} a}(k ; \kappa)\right)$ обозначим $\phi^{(j)}=\widehat{P}^{\mathscr{K}_{a}^{j}} \phi, j=0,1, \ldots, L$. Если $\mathscr{W} \in L_{w}^{p}(K), p>2$, то из леммы 2 вытекает оценка

$$
\begin{aligned}
\|\mathscr{W} \phi\| \leqslant & \sum_{j=0}^{L}\left\|\mathscr{W} \phi^{(j)}\right\| \leqslant c_{5}\|\mathscr{W}\|_{p, w} \kappa^{(n-2) / p} \sum_{j=0}^{L}\left(2^{j} a\right)^{2 / p}\left\|\phi^{(j)}\right\| \\
\leqslant & c_{5}\|\mathscr{W}\|_{p, w} \kappa^{(n-2) / p}\left(a^{2 / p}\left\|\phi^{(0)}\right\|\right. \\
& \left.+\sqrt{2} v^{1 / 2}(K) \sum_{j=1}^{L}\left(2^{j} a\right)^{2 / p-1 / 2}\left(\sum_{N \in \mathscr{K}_{a}^{j}} G_{N}\left|\phi_{N}\right|^{2}\right)^{1 / 2}\right) .
\end{aligned}
$$

Положив $n=3, p=3$ и учитьвая, что $2^{j} a<2^{j-L-2} \kappa, j=1, \ldots, L$, и $G_{N}^{+} \geqslant \kappa$ для всех $N \in \Lambda^{*}$, из (17) получаем теорему 10 .

Tеорема 10. Пусть $n=3$ (и фиксирован вектор $\left.\gamma \in \Lambda \backslash\{0\}, e=|\gamma|^{-1} \gamma\right)$. Найдутся универсальная постоянная $c>0$ и число $c_{1}=c_{1}(\Lambda)>0$ такие, что для всех $\kappa \geqslant \kappa_{0}(\Lambda)>0$, всех векторов $k \in \mathbb{R}^{3}$, всех функиий $\mathscr{W} \in L_{w}^{3}(K)$ и $\phi \in \mathscr{H}\left(\mathscr{K}_{\kappa / 8}(k ; \kappa)\right)$ справедливо неравенство

$$
\|\mathscr{W} \phi\| \leqslant\|\mathscr{W}\|_{3, w}\left(c\|\phi\|_{*}+c_{1} \kappa^{1 / 3}\|\phi\|\right)
$$

Доказательство следующей теоремы содержится в [16]. 
Tеорема 11. Пусть $n=4$ (и фиксирован вектор $\left.\gamma \in \Lambda \backslash\{0\}, e=|\gamma|^{-1} \gamma\right)$. Найдутся универсальная постоянная $c>0$ и число $c_{1}=c_{1}(\Lambda)>0$ такие, что для любой функции $\mathscr{W} \in L_{w}^{4}(K)$ существует последовательность $\kappa=\kappa_{\nu} \geqslant \kappa_{0}(\Lambda) \geqslant 2$, $\nu \in \mathbb{N}$, для которой $\kappa_{\nu} \rightarrow+\infty$ при $\nu \rightarrow+\infty$, такая, что для всех чисел $\kappa=\kappa_{\nu}$, $\nu \in \mathbb{N}$, всех векторов $k \in \mathbb{R}^{4}$ и всех функиий $\phi \in \mathscr{H}\left(\mathscr{K}_{\kappa / 8}(k ; \kappa)\right)$ справедливо неравенство

$$
\|\mathscr{W} \phi\| \leqslant\|\mathscr{W}\|_{4, w}\left(c\|\phi\|_{*}+c_{1} \kappa^{3 / 8}(\ln \kappa)^{1 / 4}\|\phi\|\right) .
$$

Пусть $b, b^{\prime} \in(0, \kappa / 2)$. Для всех $N \in \Lambda^{*}$ обозначим через $S_{b, b^{\prime}}(N)=S_{b, b^{\prime}}(k, \kappa ; N)$ число векторов $N^{\prime} \in \mathscr{K}_{b^{\prime}}$, для которых $N^{\prime}-N \in \mathscr{K}_{b}$. Положим $N_{\|}=(N, e) e$. Если $2 \pi\left|N_{\|}\right| \geqslant b+b^{\prime}$ или $2 \pi\left|N-N_{\|}\right| \geqslant 2 \kappa+b+b^{\prime}$, то $S_{b, b^{\prime}}(N)=0$.

Лемма 3. Пусть $n \geqslant 5$. Найдутся числа $\kappa_{0}=\kappa_{0}(n, \Lambda)>8 a u c_{7}=c_{7}(n)>0$ такие, что для всех $\kappa \geqslant \kappa_{0}, b, b^{\prime} \in[a, \kappa / 4), k \in \mathbb{R}^{n}, \mathcal{V} \in L^{2}(K)$ u $\phi \in L^{2}(K)$ справедливо неравенство

$$
\left\|\widehat{P}^{\mathscr{K}_{b^{\prime}} \mathscr{V} \phi^{\mathscr{K}_{b}}}\right\| \leqslant c_{7}\left(b b^{\prime} \min \left\{b, b^{\prime}\right\}\right)^{1 / 2} \kappa^{(n-3) / 2}\|\mathscr{V}\|_{2}\left\|\phi^{\mathscr{K}_{b}}\right\| .
$$

ДокАЗАтЕльство. Так как норма ограниченного оператора в $L^{2}(K)$ совпадает с нормой сопряженного к нему оператора, при доказательстве леммы 3 можно считать, что $b \leqslant b^{\prime}$. Для векторов $x \in \mathbb{R}^{n}$, для которых $x \neq(x, e) e$, обозначим

$$
\tilde{e}(x)=|x-(x, e) e|^{-1}(x-(x, e) e) \in S_{n-2}(e)
$$

(где $\left.e=|\gamma|^{-1} \gamma\right)$.

Существует число $\epsilon=\epsilon(n) \in(0,1)$ такое, что для любого $r \in(a, \kappa]$ найдутся непересекаюшиеся множества $\Omega^{(\lambda ; r)} \in S_{n-2}(e)$ и векторы $e^{(\lambda ; r)} \in \Omega^{(\lambda ; r)}, \lambda=1, \ldots, \lambda_{0}$ (где $\left.\lambda_{0}=\lambda_{0}(n, r) \in \mathbb{N}\right)$, такие, что $S_{n-2}(e)=\bigcup_{\lambda} \Omega^{(\lambda ; r)},\left|\widetilde{e}-e^{(\lambda ; r)}\right| \leqslant r \kappa^{-1}$ для всех $\tilde{e} \in \Omega^{(\lambda ; r)}$ и каждый вектор $e^{\prime} \in S_{n-2}(e)$ принадлежит не более $n-1$ множествам $\left\{\tilde{e} \in S_{n-2}(e):\left|\tilde{e}-e^{(\lambda ; r)}\right|<(1+\epsilon) r \kappa^{-1}\right\}$.

Обозначим $\mathscr{K}_{b}^{(\lambda ; r)}=\left\{N \in \mathscr{K}_{b}: \tilde{e}(k+2 \pi N) \in \Omega^{(\lambda ; r)}\right\}$. Если $N \in \mathscr{K}_{b}$, то для всех $N^{\prime} \in \Lambda^{*}$, для которых $2 \pi\left|N^{\prime}-N_{\|}^{\prime}\right| \leqslant \frac{1}{4} \epsilon r$, имеем $\left|\tilde{e}\left(k+2 \pi\left(N+N^{\prime}\right)\right)-\tilde{e}(k+2 \pi N)\right|<\epsilon r \kappa^{-1}$. Поэтому для всех функций $\mathscr{V}^{\prime} \in L^{2}(K)$, для которых $\mathfrak{V}_{N}^{\prime}=0$ при $2 \pi\left|N-N_{\|}\right|>\frac{1}{4} \epsilon r$, получаем

$$
\left\|\widehat{P}^{\mathscr{K}_{b^{\prime}} \mathscr{V}^{\prime}} \widehat{P}^{\mathscr{K}_{b}} \phi\right\|^{2} \leqslant(n-1)^{2} \sum_{\lambda}\left\|\widehat{P}^{\mathscr{K}_{b^{\prime}} \mathscr{V}^{\prime}} \widehat{P}^{\mathscr{K}_{b}^{(\lambda ; r)}} \phi\right\|^{2} .
$$

Обозначим через $S_{b, b^{\prime}}^{(\lambda ; r)}(N)$, где $N \in \Lambda^{*}$, число векторов $N^{\prime} \in \mathscr{K}_{b^{\prime}}$ таких, что $N^{\prime}-N \in$ $\mathscr{K}_{b}^{(\lambda ; r)} ; S_{b, b^{\prime}}^{(\lambda ; r)}(N) \leqslant S_{b, b^{\prime}}(N)$. Для $r \in(a, \kappa]$ и $b, b^{\prime} \in[a, \kappa / 4), b \leqslant b^{\prime}$, справедлива оценка

$$
S_{b, b^{\prime}}^{(\lambda ; r)}(N) \leqslant c_{8} v(K) b^{2} r^{n-3} \min \left\{r, b^{\prime}\left(b^{\prime}+2 \pi\left|N-N_{\|}\right|\right)^{-1} \kappa\right\},
$$

где $c_{8}=c_{8}(n)>0$, из которой следует, что для всех $\kappa>8 a, b, b^{\prime} \in[a, \kappa / 4), b \leqslant b^{\prime}$, $r \in(a, \kappa], \lambda \in\left\{1, \ldots, \lambda_{0}\right\}, \mathscr{V}^{\prime \prime} \in L^{2}(K)$ и $\phi \in L^{2}(K)$

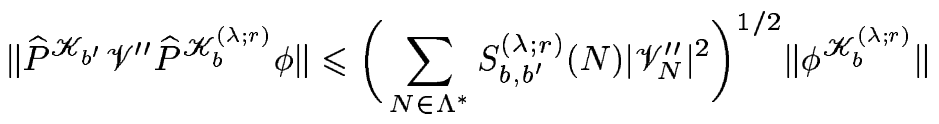

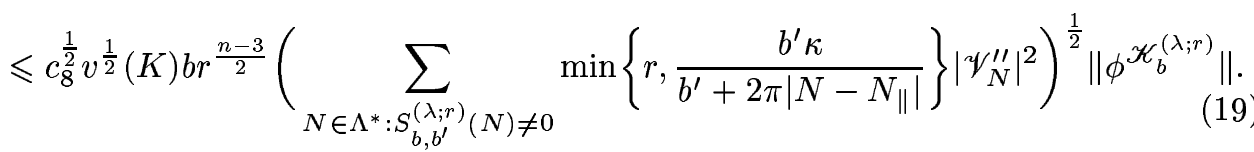


Будем далее предполагать, что $\kappa \geqslant \kappa_{0}>8 a \epsilon^{-1}$. Выберем число $J \in \mathbb{N}$, исходя из неравенств $2^{-J-3} \epsilon \kappa \leqslant a<2^{-J-2} \epsilon \kappa$. Пусть

$$
\begin{gathered}
\mathscr{A}^{\prime}=\left\{N \in \Lambda^{*}: S_{b, b^{\prime}}(N) \neq 0 \text { и } 2 \pi\left|N-N_{\|}\right|>\frac{1}{4} \epsilon \kappa\right\}, \\
\widetilde{\mathscr{A}}=\left\{N \in \Lambda^{*}: S_{b, b^{\prime}}(N) \neq 0 \text { и } 2 \pi\left|N-N_{\|}\right| \leqslant 2^{-J-2} \epsilon \kappa\right\},
\end{gathered}
$$

$\mathscr{A}_{j}=\left\{N \in \Lambda^{*}: S_{b, b^{\prime}}(N) \neq 0\right.$ и $\left.2^{-j-2} \epsilon \kappa<2 \pi\left|N-N_{\|}\right| \leqslant 2^{-j-1} \epsilon \kappa\right\}, j=1, \ldots, J$.

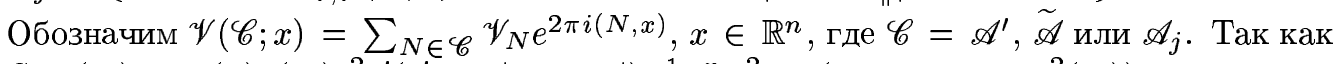
$S_{b, b^{\prime}}(N) \leqslant c_{9}(n) v(K) b^{2} b^{\prime}\left(b^{\prime}+2 \pi\left|N-N_{\|}\right|\right)^{-1} \kappa^{n-2}$, то (для всех $\left.\phi \in L^{2}(K)\right)$ справедлива оценка

$$
\begin{aligned}
\| \widehat{P}^{\mathscr{K}_{b^{\prime}} \mathscr{V}\left(\mathscr{A}^{\prime} ; \cdot\right) \widehat{P}^{\mathscr{K}_{b}} \phi \|} & \leqslant\left(\sum_{N \in \Lambda^{*}: 2 \pi\left|N-N_{\|}\right|>\frac{1}{4} \epsilon \kappa} S_{b, b^{\prime}}(N)\left|\mathscr{V}_{N}\right|^{2}\right)^{1 / 2}\left\|\phi^{\mathscr{K}_{b}}\right\| \\
& \leqslant c_{10} b\left(b^{\prime}\right)^{1 / 2} \kappa^{(n-3) / 2}\|\mathscr{V}\|_{2}\left\|\phi^{\mathscr{K}_{b}}\right\|,
\end{aligned}
$$

где $c_{10}=2 \epsilon^{-1 / 2} c_{9}(n)>0$. Для всех $j=1, \ldots, J$, полагая $r=r_{j}=2^{-j+1} \kappa$ (тогда $2 \pi\left|N-N_{\|}\right| \leqslant \frac{1}{4} \epsilon r_{j}$ для всех $N \in \mathscr{A}_{j}$, из (18) и (19) получаем оценку

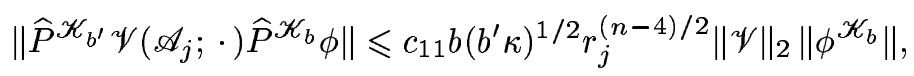

где $c_{11}=2 \sqrt{2}(n-1) \epsilon^{-1 / 2} c_{9}(n)>0$. Наконец,

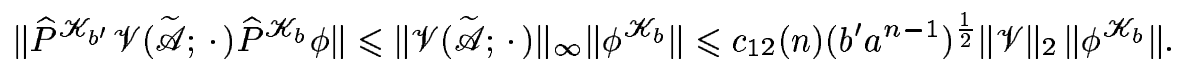

Доказьваемая оценка следует из (20)-(22). Лемма доказана.

Tеорема 12. Пусть $n=5$ (и фиксирован вектор $\left.\gamma \in \Lambda \backslash\{0\} ; e=|\gamma|^{-1} \gamma\right)$. Найдутся универсальная постоянная $c>0$ и число $c_{1}=c_{1}(\Lambda)>0$ такие, что для всех $\kappa \geqslant \kappa_{0}=\kappa_{0}(\Lambda)>0$, всех векторов $k \in \mathbb{R}^{5}$, всех функиий $\mathscr{W} \in L_{w}^{5}(K) u$ $\phi \in \mathscr{H}\left(\mathscr{K}_{\kappa / 8}(k ; \kappa)\right)$ справедливо неравенство

$$
\|\mathscr{W} \phi\| \leqslant\|\mathscr{W}\|_{5, w}\left(c\|\phi\|_{*}+c_{1} \kappa^{2 / 5}\|\phi\|\right) .
$$

Теорема 13. Пусть $n \geqslant 6$ (и фиксирован вектор $\left.\gamma \in \Lambda \backslash\{0\} ; e=|\gamma|^{-1} \gamma\right)$. Найдется постоянная $c=c(n)>0$ такая, что для всех $\kappa \geqslant \kappa_{0}=\kappa_{0}(n, \Lambda)>0$, всех векторов $k \in \mathbb{R}^{n}$, всех функиий $\mathscr{W} \in L_{w}^{2(n-3)}(K)$ и $\phi \in \mathscr{H}\left(\mathscr{K}_{\kappa / 8}(k ; \kappa)\right)$ справедливо неравенство

$$
\|\mathscr{W} \phi\| \leqslant c a^{-(n-6) /(2(n-3))}\|\mathscr{W}\|_{2(n-3), w}\left(\|\phi\|_{*}+(a \kappa)^{1 / 2}\|\phi\|\right)
$$


ДоКАЗАТЕЛЬСТВо ТЕОРЕм 12 и 13. Пусть $\mathscr{W} \in L_{w}^{p}(K), p \geqslant n, k \in \mathbb{R}^{n}(n \geqslant 5)$, $\kappa \geqslant \kappa_{0}>8 a$. Как и при доказательстве теоремы 10 , выберем число $L=L(\kappa) \in \mathbb{N}$ так, что $\kappa / 8 \leqslant 2^{L} a<\kappa / 4$, и определим для функции $\phi \in \mathscr{H}\left(\mathscr{K}_{\kappa / 8}\right) \subset \mathscr{H}\left(\mathscr{K}_{2^{L}}\right)$ функции $\phi^{(j)}=\phi^{\mathscr{K}_{a}^{j}}, j=0,1, \ldots, L$ (см. (16)). Обозначим $\mathscr{V}=|\mathscr{W}|^{2}, \mathscr{V} \in L_{w}^{p / 2}(K)$, $\|\mathscr{V}\|_{p / 2, w}=\|\mathscr{W}\|_{p, w}^{2}$. Пусть $s>0$ и $\mathscr{V}(1, s)(x)=\mathscr{V}(x)$, если $\mathscr{V}(x) \leqslant s$, и $\mathscr{V}^{(1, s)}(x)=0$ в противном случае, $x \in K ; \mathscr{V}^{(2, s)}(\cdot)=\mathscr{V}(\cdot)-\mathscr{V}(1, s)(\cdot)$. Из леммы 3 (при выборе подходящего числа $\kappa_{0}>0$ и при $\left.\kappa \geqslant \kappa_{0}\right)$ следует, что для всех $j, l=0,1, \ldots, L$ справедливо неравенство

$$
\left|\left(\phi^{(l)}, \mathscr{V}^{(2, s)} \phi^{(j)}\right)\right| \leqslant c_{7} a^{3 / 2} 2^{(j+l+\min \{j, l\}) / 2} \kappa^{(n-3) / 2}\left\|\mathscr{V}^{(2, s)}\right\|_{2}\left\|\phi^{(l)}\right\|\left\|\phi^{(j)}\right\|
$$

С другой стороны, $\left|\left(\phi^{(l)}, \mathscr{V}^{(1, s)} \phi^{(j)}\right)\right| \leqslant s\left\|\phi^{(l)}\right\|\left\|\phi^{(j)}\right\|$. Поэтому с помощью леммы 1 , полагая $s=a^{6 / p} 2^{2(j+l+\min \{j, l\}) / p} \kappa^{2(n-3) / p}\|\mathscr{V}\|_{p / 2, w}$ (при $\|\mathscr{V}\|_{p / 2, w} \neq 0$ ), получаем

$$
\left|\left(\phi^{(l)}, \mathscr{V} \phi^{(j)}\right)\right| \leqslant c_{13} a^{6 / p} 2^{2(j+l+\min \{j, l\}) / p} \kappa^{2(n-3) / p}\|\mathscr{V}\|_{p / 2, w}\left\|\phi^{(l)}\right\|\left\|\phi^{(j)}\right\|
$$

где $c_{13}=1+c_{7} c_{4}^{1 / 2}(p / 2)$. Следовательно,

$$
\begin{aligned}
\|\mathscr{W} \phi\| & \leqslant \sum_{j=0}^{L}\left\|\mathscr{W} \phi^{(j)}\right\| \\
& \leqslant c_{13}^{1 / 2}\|\mathscr{W}\|_{p, w}\left(a^{3 / p} \kappa^{(n-3) / p}\left\|\phi^{(0)}\right\|+\sqrt{2} \sum_{j=0}^{L}\left(2^{j} a\right)^{3 / p-1 / 2} \kappa^{(n-3) / p-1 / 2}\left\|\phi^{(j)}\right\|_{*}\right) .
\end{aligned}
$$

Теоремы 12 и 13 при $n \neq 6$ непосредственно вытекают из полученной оценки при $p=$ $2 p(n)$. Пусть $n=p=6$. Из (23) имеем

$$
\begin{aligned}
\|\mathscr{W} \phi\|^{2} & \leqslant 2 \sum_{0 \leqslant l \leqslant j \leqslant L}\left|\left(\phi^{(l)}, \mathscr{V} \phi^{(j)}\right)\right| \\
& \leqslant c_{14}(n)\|\mathscr{V}\|_{3, w}\left(a \kappa\left\|\phi^{(0)}\right\|^{2}+\|\phi\|_{*}^{2}+\sum_{1 \leqslant l \leqslant j \leqslant L} 2^{-(j-l) / 6}\left\|\phi^{(l)}\right\|_{*}\left\|\phi^{(j)}\right\|_{*}\right) \\
& \leqslant c_{15}\|\mathscr{V}\|_{3, w}\left(a \kappa\left\|\phi^{(0)}\right\|^{2}+\|\phi\|_{*}^{2}\right),
\end{aligned}
$$

где $c_{15}=2\left(1-2^{-1 / 6}\right)^{-1} c_{14}(n)$. Из последнего неравенства следует теорема 13 при $n=6$. Теоремы 12 и 13 доказаны.

Теорема 5 вытекает из теорем 9-13, если принять во внимание оценку $\|\phi\|^{2} \leqslant|\gamma| \times$ $(2 \pi \kappa)^{-1}\|\phi\|_{*}^{2}$, которая справедлива при всех $\kappa>0, k \in \mathbb{R}^{n}:(k, \gamma)=\pi$ и $\phi \in \widetilde{H}^{1}(K)$, и учесть, что $\|\mathscr{W}\|_{n, w}^{(\infty)}=0$, если $\mathscr{W} \in L_{w}^{p}(K), p>n \geqslant 3$. 


\section{СПИСОК ЦИТИРОВАННОЙ ЛИТЕРАТУРЫ}

[1] Thomas L. E. Time-dependent approach to scattering from impurities in a crystal // Comm. Math. Phys. 1973. V. 33. P. 335-343.

[2] Рид М., Саймон Б. Методы современной математической физики. Т. 4. Анализ операторов. М.: Мир, 1982.

[3] Бирман М.Ш., Суслина Т. А. Абсолютная непрерывность двумерного периодического магнитного гамильтониана с разрьвным векторным потенциалом // Алгебра и анализ. 1998. T. 10. № 4. C. 1-36.

[4] Sobolev A. Absolute Continuity of the Periodic Magnetic Schrödinger Operator. Res. Report no. 97/06: Univ. of Sussex, 1997; Preprint ESI № 495. Wien: Erwin Schrödinger Internat. Institute for Math. Phys., 1997.

[5] Бирман М.Ш., Суслина Т. А. Периодический магнитньй гамильтониан с переменной метрикой. Проблема абсолютной непрерьвности // Алгебра и анализ. 1999. Т. 11. № 2. C. 1-40.

[6] Kuchment P., Levendorskiř S. On absolute continuity of spectra of periodic elliptic operators // Oper. Theory Adv. Appl. 1999. V. 108. P. 291-297.

[7] Shen Z. On Absolute Continuity of the Periodic Schrödinger Operators. Preprint ESI № 597. Wien: The Erwin Schrödinger Internat. Institute for Math. Phys., 1998; Preprint \# 99-189: Texas Math. Physics Archive, 1999.

[8] Morame A. Absence of singular spectrum for a perturbation of a two-dimensional LaplaceBeltrami operator with periodic electro-magnetic potential // J. Phys. A: Math. Gen. 1998. V. 31. P. 7593-7601.

[9] Morame A. The Absolute Continuity of the Spectrum of the Maxwell Operator in Periodic Media. Preprint \# 99-308: Texas Math. Physics Archive, 1999.

[10] Данилов Л. И. Оценки резольвенты и спектр оператора Дирака с периодическим потенциалом // ТМФ. 1995. Т. 103. №1. С. 3-22.

[11] Данилов Л. И. Абсолютная непрерывность спектра периодического оператора Дирака // Дифференц. уравнения. 2000. Т. 36. № 2. С. 233-240.

[12] Данилов Л.И. О спектре двумерного периодического оператора Дирака // ТМФ. 1999. Т. 118. №1. С. 3-14.

[13] Данилов Л. И. Спектр оператора Дирака с периодическим потенциалом. III. Деп. ВИНИТИ № 2252-В92 (10.07.92). Ижевск: ФТИ УрО РАН, 1992.

[14] Birman M.Sh., Suslina T.A. The Periodic Dirac Operator is Absolutely Continuous. Preprint ESI № 603. Wien: Erwin Schrödinger Internat. Institute Math. Phys., 1998.

[15] Данилов Л.И. О спектре периодического оператора Дирака // ТМФ. 2000. Т. 124. № 1. С. 3-17.

[16] Данилов Л. И. Об абсолютной непрерывности спектра периодических операторов Шрёдингера и Дирака. І. Деп. ВИНИТИ № 1683-В00 (15.06.00). Ижевск: ФТИ УрО РАН, 2000.

[17] Kuchment P. Floquet Theory for Partial Differential Equations. Basel: Birkhäuser Verlag, 1993.

[18] Рид М., Саймон Б. Методы современной математической физики. Т. 2. Гармонический анализ. Самосопряженность. М.: Мир, 1978.

[19] Стейн И. Сингулярные интегралы и дифференциальные свойства функций. М.: Мир, 1973.

Физико-технический институт Удмуртского научного центра Уро РАН, г. Ижевск E-mail: lyu@otf.fti.udmurtia.su 